\title{
A Theoretical Study of CCD4a Dioxygenase of Citrus, a Cleavage Enzyme of Carotenoids in Plants
}

\author{
Mauricio Vega-Teijido, Margot P. Zunini, Carolina López \& Maria J. Rodrigo
}

\section{Introduction}

The coloration in citrus and other plants is due the accumulation of carotenoids, which are isoprenoid pigments of 40 carbon atoms (C40) and its derivatives. The enzymatic function of CCD4a has been associated previously with the metabolism of carotenoids in Chrysanthemum morifolium [1]. The flowers with white petals showed a high expression of CCD4a in contrast with those with yellow petals that showed a low expression. The interpretation was that the synthetized carotenoids are degraded by CCD4a to produce colorless petals [1]. In saffron stigmas (Crocus sativus) $\mathrm{CCD} 4 \mathrm{a}$ and $\mathrm{CCD} 4 \mathrm{~b}$ were associated with the transformation of zeaxanthin in the apocarotenoids responsible by the color, taste and aroma of this so appreciated spice [2].

Recently, Rodrigo et al. [3], reported a series of CCD4type citrus dioxygenases involved in the generation of C30 apocarotenoids. These natural compounds provide an attractive reddish-orange pigmentation to the peel of many sweet oranges and mandarins, which is associated with its perception of quality. Among them, CCD4b1 is the first reported case of dioxygenase that cleaves double bonds of carotenoids $\mathrm{C} 40$ in the position 7,8 or 7'-8', generating the corresponding $\mathrm{C} 30$ derivative. In this case, the possible products are $\beta$-citraurin or 8 - $\beta$-apocarotenal depending on the carotenoid used as substrate, that is, zeaxanthin or $\beta$-carotene, respectively. $\beta$-cryptoxanthin substrate can derivate in one or other product depending on the ring cleaved. Still remains unclear the specific position where CCD4a enzymes can cleave a carotenoid double bond. Two possible double bonds could be cleaved, 7-8/7'-8' as CCD4b1 or $9-10 / 9^{\prime}-10^{\prime}$ as has been previously reported by Rubio et al. [2] for CCDD4a of Crocus sativus with zeaxanthin and $\beta$-carotene as substrates. In the case of CCD4a of citrus the specific position is not confirmed yet and this is one of the aims of our studies (Figure 1).

Here we report a new tridimensional model of CCD4a and their complexes with a series of 3 carotenoids ( 4 different complexes). The structure of CCD4a was optimized and stabilized in an explicit water box using Molecular Dynamics (MD). The complexes with the ligands were constructed by means Docking. Finally, MD was used to optimize, and then, simulate and study these molecular systems.

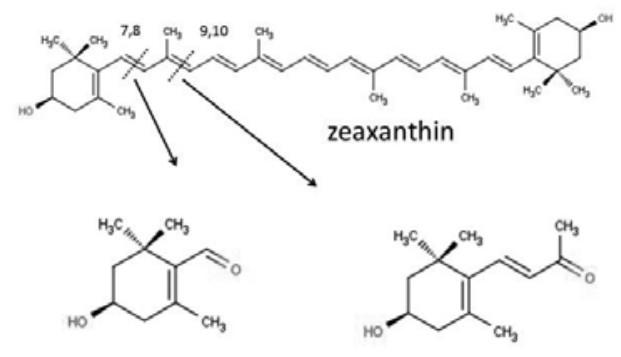

3-hydroxy- $\beta$-cyclocitral 3-hydroxy- $\beta$-ionone

Figure 1. Schematic representation of zeaxanthin and both possible subproducts of cleavage. 


\section{Methods}

The structures were modeled by sequence homology (2biw, PDB code [4]) and Molecular Dynamics (MD). After this, Docking calculations [5] were performed in CCD4a receptor with thousands of initial ligand conformations, in order to find all the possible poses in the active site. The evaluation of the interaction energy was done by means of ASE scoring posing, an Amber99 refinement and LondonDG rescoring. The force field used in MD was Amber99, with a $0.002 \mathrm{ps}$ time-step using the NPA algorithm. The water box was constructed orthorhombic with $6 \mathrm{~nm}$ distances to the faces. The MD procedure was implemented by heating of $100 \mathrm{ps}$ from 0 to $300 \mathrm{~K}, 100 \mathrm{ps}$ of equilibration and $5 \mathrm{~ns}$ of simulation at $300 \mathrm{~K}$, in order to obtain values with statistical significance.

\section{Results and Discussion}

The docking results are presented in two tables, one for the all-trans ligands (Table 1) and other for the 9-10-Z isomers (Table 2). All-trans ligands are known as substrates of CCD enzymes in general. In docking results we observed that ligands adopt a curvature in the active site, so we tested ligands with a trans-cis transformation of the 9-10 double bond. Some examples, as CCD8, can cleave cis isomers [6]. Even we can mention that in the 2 biw crystallographic structure, used as template for our model, the soaking procedure of the crystal without $\mathrm{Fe}(2+)$ (inactive enzyme) were done with the all-trans substrate. Despite this, the final structure of the ligand showed an isomerization when the active form with $\mathrm{Fe}(2+)$ was obtained [4]. The authors suggest that some members of the CCD family can act as isomerases.

Table 1. LondonDG scores for the best five solutions. In $\beta$-cryptoxanthin column two solutions correspond to the conformation with the $\mathrm{OH}$ moiety out of the active site.

\begin{tabular}{|c|c|c|c|}
\hline & zeaxanthin & $\beta$-crtyptoxanthin & $\beta$-carotene \\
\hline 1 & -20.91 & -19.94 (out) & -16.43 \\
\hline 2 & -20.62 & -19.93 & -16.25 \\
\hline 3 & -20.6 & -19.67 & -16.13 \\
\hline 4 & -20.54 & -19.63 (out) & -16.04 \\
\hline 5 & -20.53 & -19.41 & -15.83 \\
\hline
\end{tabular}

The results of docking (Table 1) show LondonDG score of $-20,-19$ and $-16 \mathrm{kcal} / \mathrm{mol}$, for all-trans zeaxanthin, $\beta$-cryptoxanthin and $\beta$-carotene, respectively (Figure 2). The correlation can be associated with the number of hydroxylic moieties (two, one or none), this correlation were experimentally observed in CCD4b1 for these substrates [3]. The same correlation was observed with the $9-10-Z$ isomers with slightly more favorable scores and only one solution with the $\mathrm{OH}$ moiety out of the active site.

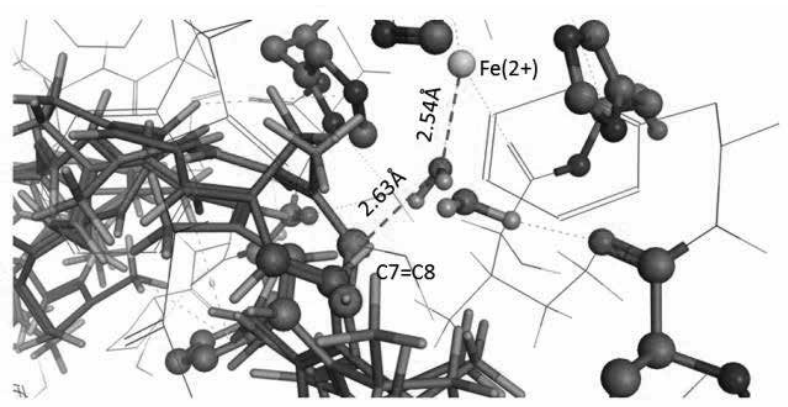

Figure 2. Ligands docked in the active site of CCD4a. One water molecule occupies one coordination site of $\mathrm{Fe}(2+)$. This site is occupied by a reactive oxygen molecule during the catalysis.

Table 2. LondonDG scores for the best five $9-10-Z$ isomers solutions. In $\beta$-cryptoxanthin column one solution corresponds to the conformation with the $\mathrm{OH}$ moiety out of the active site.

\begin{tabular}{|c|c|c|c|}
\hline & zeaxanthin & $\beta$-crtyptoxanthin & $\beta$-carotene \\
\hline 1 & -21.85 & -19.85 & -16.53 \\
\hline 2 & -21.67 & -19.65 (out) & -16.48 \\
\hline 3 & -21.13 & -19.50 & -16.42 \\
\hline 4 & -20.55 & -19.49 & -16.37 \\
\hline 5 & -20.16 & -19.39 & -16.35 \\
\hline
\end{tabular}

All the molecular complexes showed a stable behavior when were simulated by MD (Figure 3). The interaction of the ligands with the actives site of CCD4a was estimated by the Uab value that is the potential energy of interaction between the ligand and all the surrounding. The same sequence in the estimated interaction strength (Uab) was obtained for the three ligands using MD [5] (Table 3). Very similar values were observed for the two 
possible orientations of $\beta$-cryptoxanthin in the active site, so it is hard to say if one or other is a preferential substrate for CCD4a.

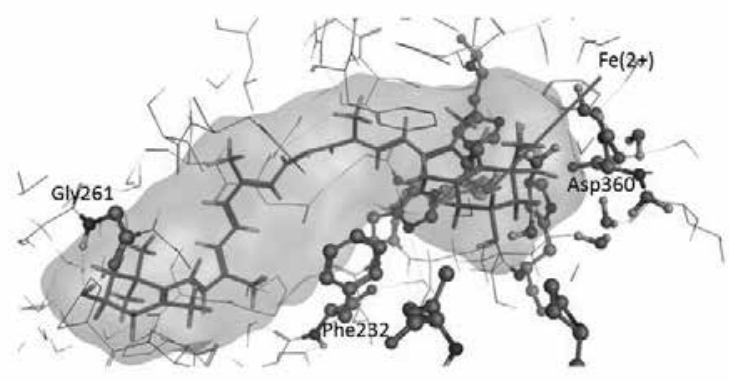

Figure 3. Zeaxanthin ligand in the active site of CCD4a.

Table 3. Average values for the Uab ( $\mathrm{kcal} / \mathrm{mol})$ and standard deviation for MD calculations.

\begin{tabular}{|l|c|}
\hline \multicolumn{1}{|c|}{ Uab } & Average (Std. Dev) \\
\hline zeaxanthin & $-126.0(4.2)$ \\
\hline$\beta$-crtyptoxanthin (OH out) & $-110.8(4.5)$ \\
\hline$\beta$-cryptoxanthin & $-107.0(5.0)$ \\
\hline$\beta$-carotene & $-87.5(4.3)$ \\
\hline
\end{tabular}

The distance between the water molecule coordinated with $\mathrm{Fe}(2+)$ and $\mathrm{C} 8$ is in a range of $2-4 \mathrm{~A}$, the same occurs for C9 (Figure 2). Both distances are alternative one shorter than the other during all the MD simulations, so, depending on this parameter both double bonds can be aim of the cleavage when an $\mathrm{O} 2$ molecule is the one coordinated to $\mathrm{Fe}(2+)$. All the process may involve other parameters influencing the selective cleavage or unselective, these questions are those we hope could be clarified in next steps of our work.

\section{Conclusions}

These findings will be discussed considering the potential in vivo substrates and products, and the physiological role in citrus fruits.

\section{Acknowledgments}

The authors are grateful for the support given for PEDECIBA, CSIC, ANII and IBERCAROT.

\section{References}

1. Ohmiya, A., Kishimoto, S., Ainda, R., Yoshioka, S., and Sumitomo, K. (2006) Plant Physiol. 142, 1193-1201.

2. Rubio-Moraga, A., Rambla, J.L., Fernández de Carmen, A., Trapero-Mozos, A., Ahrazem, O., Orzáez, D., Granell, A., Gómez-Gómez, L. 2008) J Biol Chem 283(36): 24816-24825.

3. Rodrigo, M.J., Alquézar, B., Alós, E., Medina, V., Carmona, L., Bruno, M., Al-Babili, S., Zacarías, L. (2013) Journal of Experimental Botany 64(14): 4461-4478.

4. Kloer, D.P., Ruch, S., Al-Babili, S., Beyer, P., Schulz, G.E. (2005) Science. 308, 267-269.

5. MOE. Molecular Operating Environment (MOE 2007.09), Chemical Computing Group, Inc., Montreal, Quebec, Canada.

6. Harrison, P.J., Bugg, T.H.D. (2014) Arch. Biochem. Biophys. 554, 105-111.

\section{Mauricio Vega-Teijido ${ }^{a, b}$, Margot Paulino Zuninia, Carolina López ${ }^{a}$ \& Maria J. Rodrigo ${ }^{c}$}

\author{
${ }^{a}$ CeBioinfo, DETEMA, Facultad de Química, UdelaR \\ ${ }^{\mathrm{b}}$ CCBG, DETEMA, Facultad de Química, UdelaR \\ c Laboratorio Fisiología y Biotecnología Postcosecha Instituto de \\ Agroquímica y Tecnología de Alimentos IATA-CSIC, Valencia, España.
}

